\title{
Involvement of Matrix Metalloproteinase in Neuroblast Cell Migration from the Subventricular Zone after Stroke
}

\author{
Seong-Ryong Lee, ${ }^{1,3}$ Hahn-Young Kim, ${ }^{1,4}$ Jadwiga Rogowska, ${ }^{5}$ Bing-Qiao Zhao, ${ }^{1,3}$ Pradeep Bhide, ${ }^{2}$ Jack M. Parent, ${ }^{6}$ and \\ Eng H. Lo ${ }^{1,2,7}$ \\ ${ }^{1}$ Neuroprotection Research Laboratory, Departments of Neurology and Radiology, and 2Neuroscience Center, Massachusetts General Hospital, Boston, \\ Massachusetts 02129, ${ }^{3}$ Department of Pharmacology, Keimyung University, Taegu 700-712, Korea, ${ }^{4}$ Department of Neurology, Konkuk University, Seoul \\ 143-701, Korea, ${ }^{5}$ Department of Psychiatry, McLean Hospital, Belmont, Massachusetts 02478, ${ }^{6}$ Department of Neurology, University of Michigan Medical \\ School, Ann Arbor, Michigan 48109, and 7Program in Neuroscience, Harvard Medical School, Boston, Massachusetts 02115
}

\begin{abstract}
After brain injury, neuroblast cells from the subventricular zone (SVZ) expand and migrate toward damaged tissue. The mechanisms that mediate these neurogenic and migratory responses remain to be fully dissected. Here, we show that bromodeoxyuridine-labeled and doublecortin-positive cells from the SVZ colocalize with the extracellular protease matrix metalloproteinase-9 (MMP-9) during the 2 week recovery period after transient focal cerebral ischemia in mice. Treatment with the broad spectrum MMP inhibitor GM6001 significantly decreases the migration of doublecortin-positive cells that extend from the SVZ into the striatum. These data suggest that MMPs are involved in endogenous mechanisms of neurogenic migration as the brain seeks to heal itself after injury.
\end{abstract}

Key words: neurogenesis; cerebral ischemia; extracellular matrix; stroke recovery; neural precursor; stroke

\section{Introduction}

One of the most significant paradigm shifts in neuroscience is the recognition that neurogenesis persists in adult mammalian brain (Gross, 2000). The two most widely acknowledged pockets of active neurogenesis comprise the subventricular zone (SVZ) and hippocampal subgranular zone. Accumulating data now indicate that these areas of ongoing neurogenesis dramatically respond after brain injury, expanding and contributing to streams of neuroblasts that track toward damaged brain tissue (Arvidsson et al., 2002; Parent et al., 2002; Zhang et al., 2004). An important study recently showed that neurogenesis after brain injury is a functionally meaningful response; blockade of neurogenic cell division with irradiation worsens outcomes after cerebral ischemia (Raber et al., 2004). Ultimately, the hope is that one can harness these endogenous substrates of recovery to treat a wide range of CNS disorders. To achieve this goal, a better understanding is required of the mechanisms underlying the neurogenic response to brain injury. Various growth factors have been implicated in the expansion of SVZ neurogenesis after stroke and brain trauma (Nakatomi et al., 2002; Chen et al., 2005; Schneider et al., 2005), but how these neuroblasts migrate remain to be fully understood. Recent studies suggested that upregulation of stem cell and stromal cell-derived factors in damaged brain provide the chemokine signals that attract neuroblasts (Jin et al., 2002; Sun et al., 2004; Robin et al., 2005). However, the question remains how these

Received Sept. 26, 2005; revised Jan. 16, 2006; accepted Jan. 17, 2006.

This work was supported in part by National Institutes of Health Grants R01-NS37074, R01-NS40529, R01NS48422, and P50-NS10828 and by a Bugher Stroke Award from the American Heart Association.

Correspondence should be addressed to Eng H. Lo, Neuroprotection Research Laboratory, Massachusetts General Hospital East 149-2401, Charlestown, MA 02129. E-mail: Lo@helix.mgh.harvard.edu.

D0I:10.1523/JNEUROSCI.4085-05.2006

Copyright $\odot 2006$ Society for Neuroscience $\quad$ 0270-6474/06/263491-05\$15.00/0 neuroblasts move through the extracellular tangle of damaged brain tissue.

Here, we hypothesize that neuroblasts migrate toward damaged brain by using matrix metalloproteinases (MMPs). MMPs form a family of zinc endopeptidases that can modulate all components of brain extracellular matrix (Yong et al., 2001; Lo et al., 2002; Rosenberg, 2002). MMPs are involved in matrix remodeling that permits axonal extensions and recovery after CNS injury (Larsen et al., 2003; Reeves et al., 2003). Hence, MMPs may be ideal candidates to regulate stroke-induced neuroblast migration. In this study, we use a mouse model of focal stroke to assess the role for MMPs in mediating neuroblast migration from the SVZ into damaged striatum.

\section{Materials and Methods}

Animal stroke model. Adult male CD-1 mice (Charles River, Wilmington, MA) weighing 30-40 g were used in this study. All protocols for these studies were approved by the institutional animal care and use committee according to National Institutes of Health Guidelines. Mice were allowed free access to water and food before surgery. Anesthesia was induced with $3 \%$ and maintained with $1 \%$ halothane in a mixture of $70 \%$ nitrous oxide and 30\% oxygen. Right middle cerebral artery occlusion was induced by a $7-0$ nylon monofilament insertion through the external carotid artery stump following standard procedures. After $1 \mathrm{~h}$, the filament was withdrawn to reperfuse the ischemic brain.

Bromodeoxyuridine cell labeling. Following standard procedures, intraperitoneal injections of bromodeoxyuridine (BrdU) $(50 \mathrm{mg} / \mathrm{kg}$; Roche Diagnostics, Mannheim, Germany) were given twice a day, at least $6 \mathrm{~h}$ apart, during days 5-13 after focal cerebral ischemia (Parent et al., 2002). All mice were killed at 2 weeks after focal cerebral ischemia.

MMP inhibitor injection. Intraperitoneal injections of broad-spectrum MMP inhibitor GM6001 (100 mg/kg, i.p.; Chemicon, Temecula, CA) or vehicle $(4 \%[\mathrm{w} / \mathrm{v}]$ carboxymethylcellose in water) was given once daily, during days 3-12 (10 consecutive days) after focal cerebral ischemia. To 
exclude the possibility of neuroprotective effect of MMP inhibitor, we started the injection of GM6001 on the third day after ischemia.

Confocal immunohistochemistry. Mice were killed by an overdose of sodium pentobarbital. After transcardiac perfusion with PBS and 4\% ice-cold phosphate-buffered paraformaldehyde, brains were fixed overnight and precipitated in $30 \%$ sucrose solution, and frozen coronal sections ( $20 \mu \mathrm{m}$ thick) were prepared using a cryostat. Single and double-label immunofluorescence histochemistry was performed. Primary antibody dilutions used were 1:200 for BrdU (mouse monoclonal; Roche Diagnostics), 1:500 for doublecortin (DCX) (from the J. M. Parent laboratory), and 1:200 for MMP-9 (rabbit monoclonal; gift from Dr. Robert Senior, Washington University, St. Louis, MO). For BrdU immunostaining, DNA was denatured by incubating the tissue section in $2 \mathrm{~N} \mathrm{HCl}$ for 30 min at $37^{\circ} \mathrm{C}$ followed by a $10 \mathrm{~min}$ wash in $0.1 \mathrm{M}$ boric acid solution, $\mathrm{pH}$ 8.5. Secondary antibodies used for immunofluorescence were goat antirabbit IgG conjugated to FITC (1:200; Jackson ImmunoResearch Laboratories, West Grove, PA) or tetramethylrhodamine isothiocyanate (TRITC) (1:200; Jackson ImmunoResearch), and goat anti-mouse IgG conjugated to FITC or TRITC. Images were obtained using a Zeiss (Thornwood, NY) LSM confocal laser microscope as single optical images or $z$-series stacks.

Image analysis. To quantify effects of MMP inhibition on DCX signals, NIH ImageJ was used to process tiff images. Three image processing steps were performed. First, a 10-pixel-wide rolling-ball subtraction algorithm was used to remove background noise (Sternberg, 1983). Second, a two-pixel-wide median filter was used to suppress pixel noise. Image quality was visually assessed using a three-dimensional surface plot. Finally, a cutoff of mean plus 5 SDs was used to automatically threshold and calculate DCXpositive pixel numbers for five sections per brain, all centered in the SVZ axial regions. A Student's $t$ test was used to assess differences in DCXpositive pixel counts between vehicle and MMPinhibited (GM6001-treated) brains. $p<0.05$ was considered significant.

\section{Results}

Mice were subjected to focal cerebral ischemia by transiently occluding their middle cerebral arteries for $60 \mathrm{~min}$. Beginning at $5 \mathrm{~d}$ after stroke, mice were injected intraperitoneally with BrdU twice daily for $8 \mathrm{~d}$. One day after cessation of BrdU injections (14 d after stroke onset), brains were removed for analysis. As expected, the ischemic hemisphere showed an expanded SVZ with increased BrdU labeling (Fig. $1 a-c$ ). This ipsilateral BrdU response was associated with an increase in the density of DCX-positive neuroblasts compared with the contralateral side (Fig. 1d). Z-stack confocal analysis demonstrated that areas of elevated BrdU signal also coincided with streams of DCX-positive
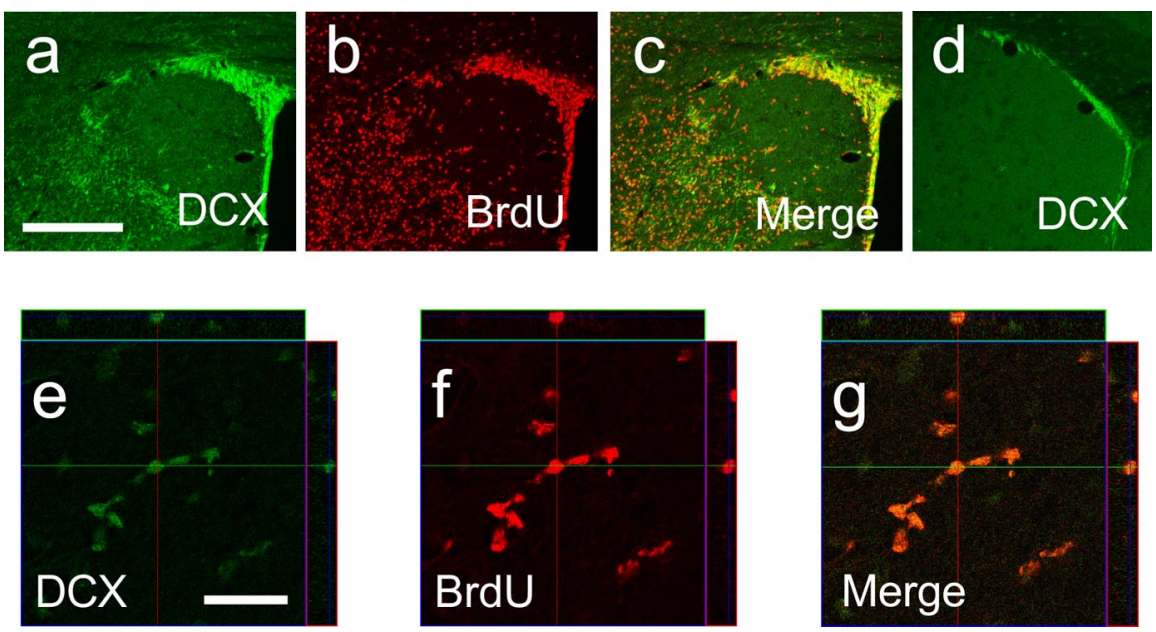

Figure 1. Neurogenic response originating from the subventricular zone (SVZ) at 2 weeks after focal stroke in mouse brain. $\boldsymbol{a}-\boldsymbol{c}$, As expected, BrdU labeling in the ischemic hemisphere coincided with an expanded SVZ and DCX-positive neuroblasts that appear to migrate toward damaged striatum. $\boldsymbol{d}$, DCX levels were low in contralateral SVZ and striatum. $\boldsymbol{e}$ - $\boldsymbol{g}$, Z-stack confocal microscopy confirms double labeling of BrdU-positive cells with the migrating neuroblast marker DCX.
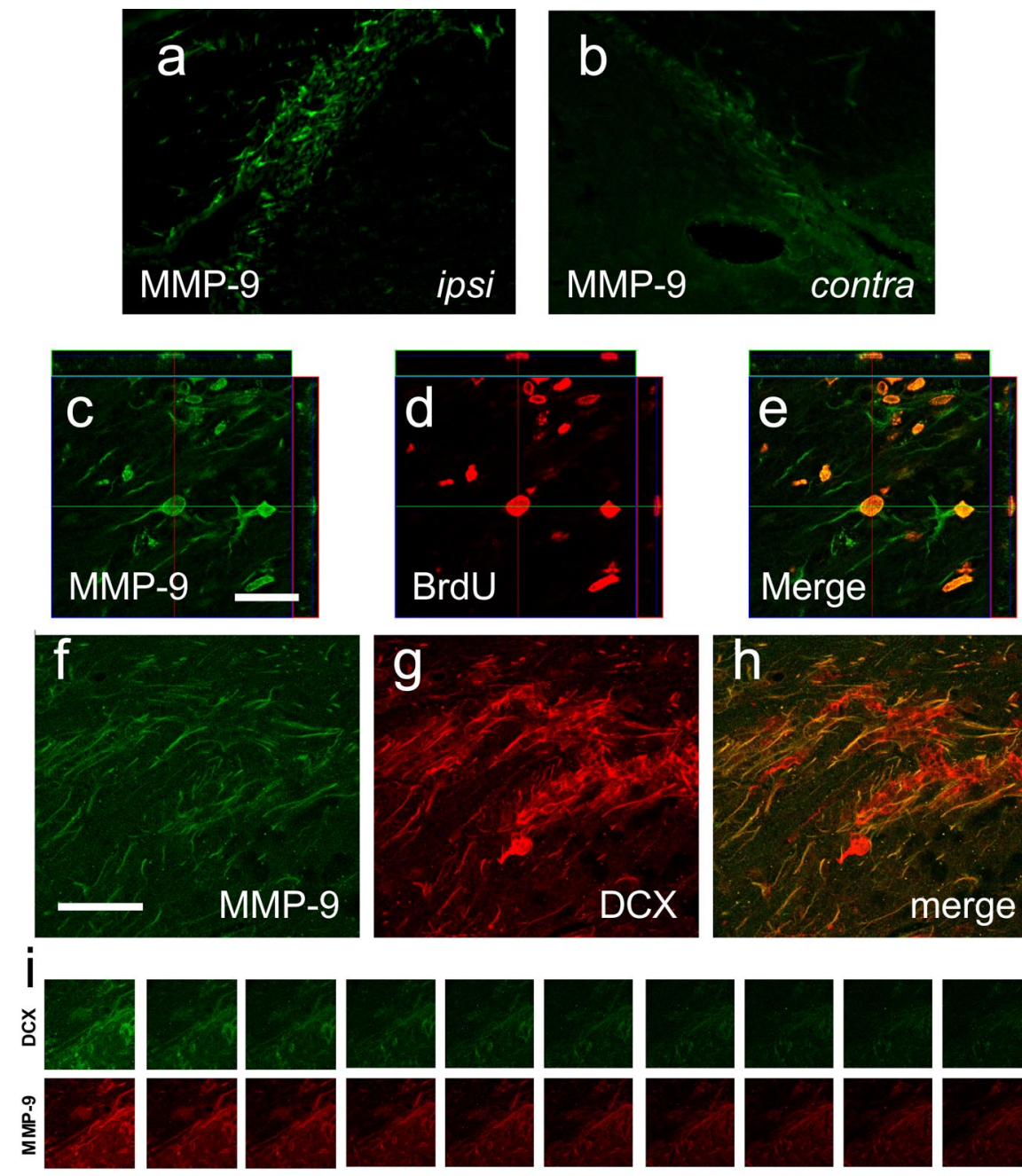

merge
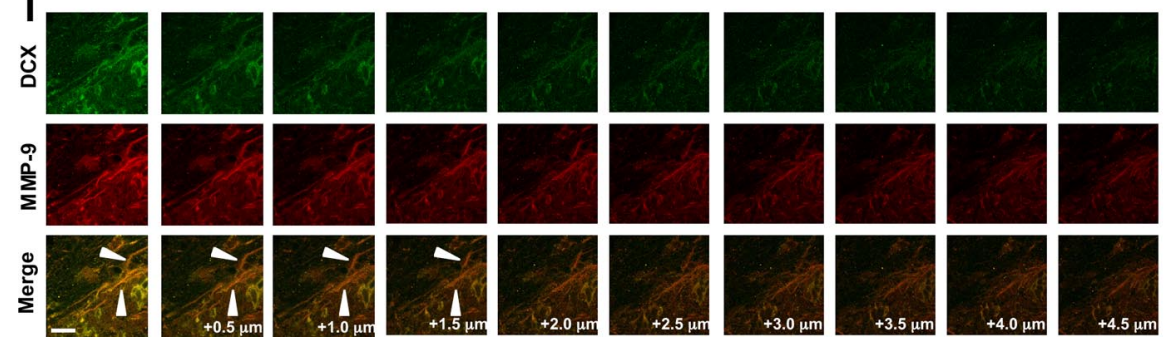

Figure 2. $\quad \boldsymbol{a}, \boldsymbol{b}, \mathrm{MMP}-9$ levels in the ischemic hemisphere were higher than the contralateral side. $\boldsymbol{c}-\boldsymbol{e}, \mathbf{Z}$-stack confocal images demonstrate colocalization of MMP- 9 with BrdU within the same cells. $\boldsymbol{f}-\boldsymbol{h}$, Within filamentous processes of DCX labeling, MMP-9 is also expressed. $i$, Consecutive confocal image planes show colocalization of MMP-9 with DCX-positive cellular structures. contra, Contralateral nonischemic side; ipsi, ipsilateral ischemic side. 

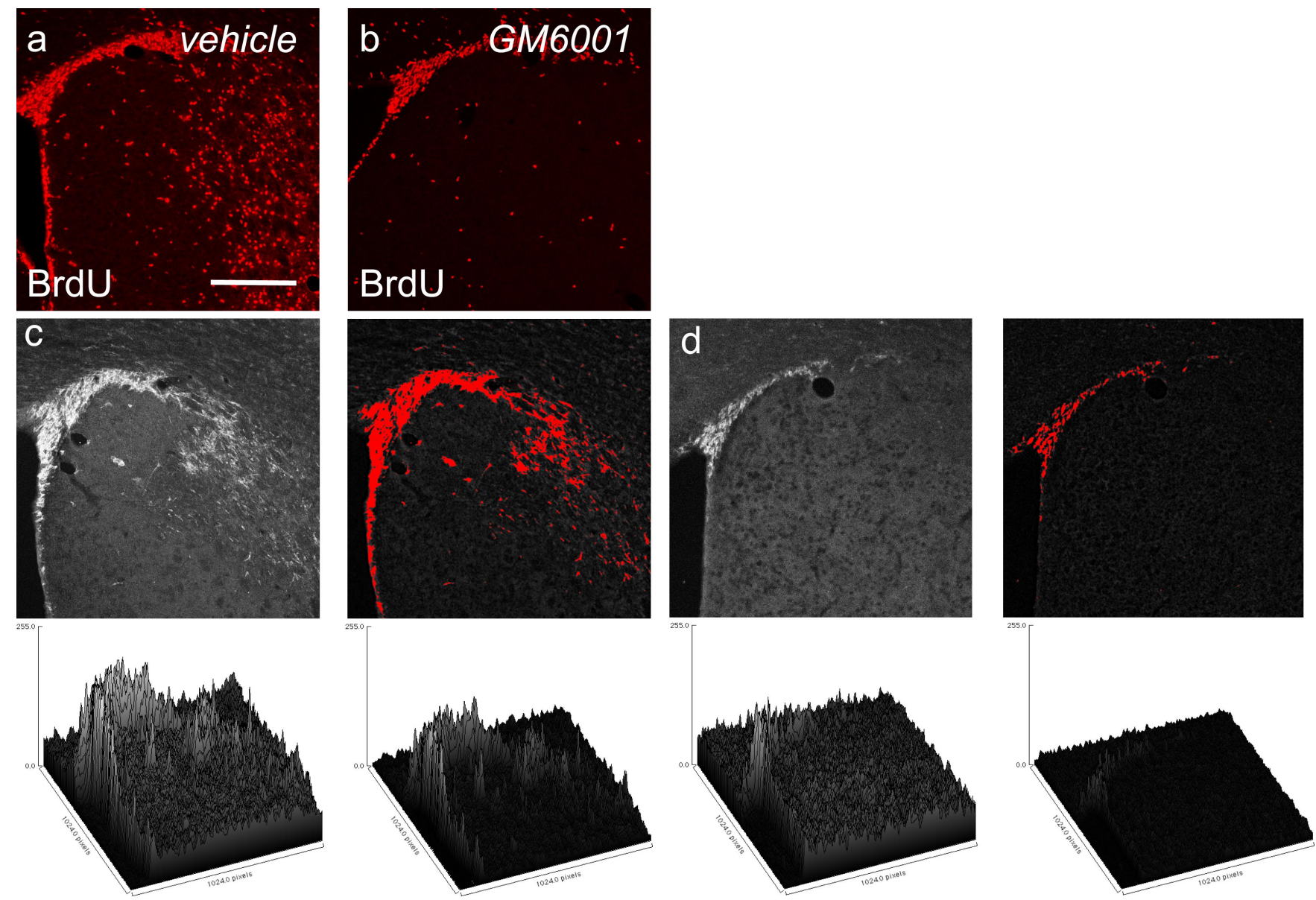

Figure 3. Suppression of DCX-positive neuroblast migration after stroke by MMP inhibition. Mice treated with the MMP inhibitor GM6001 were compared against vehicle controls. $\boldsymbol{a}$, In vehicle controls, BrdU labeling was increased in ischemic SVZ and striatum. $\boldsymbol{b}$, BrdU labeling was decreased in GM6001-treated mice. $\boldsymbol{c}$, Representative section from a control brain after stroke shows SVZ expansion and migration of DCX-positive cells toward damaged striatum (top left image) Surface plots of DCX signals demonstrate the image-processing technique used for quantitative data analysis. The initial surface plot shows significant noise (bottom left panel). Subtraction and median filtering removes background and pixel noise to reveal cleaner profiles of DCX migration (bottom right panel). Final thresholded image demonstrates pixel areas (in red) that are identified as DCX-positive signals (top right image). $\boldsymbol{d}$, Representative images and surface plots from a GM6001treated mouse show thresholded images (top) and surface plots (bottom) of reduced DCX migration.

neuroblast cells that appeared to laterally migrate from the SVZ toward the damaged striatum within the ischemic territory (Fig. $1 e-g$ ).

Because we have shown previously that among the large MMP family of extracellular proteases, MMP-9 was the most responsive to acute brain injury (Wang et al., 2000; Asahi et al., 2001; Wang et al., 2003), we sought evidence to implicate this extracellular matrix protease in our model of stroke-induced neuroblast migration. MMP-9 levels within the SVZ were higher in the ischemic hemisphere compared with the contralateral side (Fig. $2 a, b)$. Z-stack confocal analysis of double-stained immunohistochemistry demonstrated that MMP-9 was colocalized in cells that incorporated BrdU (Fig. $2 c-e$ ) and streams of DCX-positive structures that extended from the SVZ into damaged striatal tissue in the ischemic hemisphere (Fig. $2 f-i$ ).

Although the colocalization of MMP-9 with these markers of neuroblast migration after stroke supports our central idea, it does not prove causality. To further test our hypothesis, we next examined mice subjected to $60 \mathrm{~min}$ focal cerebral ischemia, and then treated with either vehicle (controls; $n=6$ ) or $100 \mathrm{mg} / \mathrm{kg}$ of the broad spectrum MMP inhibitor GM6001 (100 mg/kg, i.p.; $n=6$ ) daily from days 3-12 (10 consecutive days) after stroke. Treatment was begun on day 3 to exclude potential neuroprotective effects of MMP inhibition during the acute stages of stroke.
Our data showed that MMP inhibition clearly suppressed the neurogenic migratory response induced by stroke. BrdU labeling at $14 \mathrm{~d}$ was lower in GM6001-treated mice versus vehicle controls (Fig. $3 a, b)$. The extension of DCX-positive neuroblasts from the SVZ into damaged brain areas was also markedly reduced by MMP inhibition (Fig. $3 c, d$ ). Because DCX-positive structures were extensive and sometimes intertwined, precise counting of individual cells was difficult. Hence, we used an image-processing algorithm to compute pixel areas of DCX staining instead. Briefly, a rolling-ball subtraction algorithm and a median filter was used to remove background and pixel noise, respectively. These image-processing steps reduced noise and allowed us to more reliably define "hills" that represented DCXpositive signals (Figs. $3 c, d$ ). A standard $t$ test then demonstrated that DCX-positive signal areas were significantly reduced in mice treated with the MMP inhibitor (17,210 \pm 2095 pixels) compared with control mice $(50,001 \pm 6787$ pixels; mean \pm SD; $n=6$ per group; $p<$ $0.001)$.

\section{Discussion}

Under normal conditions, the SVZ is an active area of persistent adult neurogenesis in the rodent brain, and neuroblasts migrate along the sagittal axis of the rostral migratory stream to populate the olfactory bulb. However, after stroke and other forms of brain injury, neuroblasts swerve away from their designated path and mi- 
grate toward damaged tissue instead (Lichtenwalner and Parent, 2005; Zhang et al., 2005). Cell migration requires an active extracellular protease response. In the present study, our data show that MMPs are colocalized with BrdU and DCX-positive neuroblasts, and inhibition of MMPs significantly suppresses the movement of these neuroblasts from the SVZ into damaged tissue. These findings demonstrate that the MMP family of extracellular proteases plays a key role in mediating the neurogenic and migratory response of SVZ neuroblasts after stroke and brain injury.

The role of MMPs in brain injury has been investigated extensively. MMPs are rapidly upregulated after brain injury after trauma, ischemia, and hemorrhage (Mun-Bryce and Rosenberg, 1998; Gasche et al., 1999; Heo et al., 1999; Wang et al., 2000; Power et al., 2003). Degradation of neurovascular substrates lead to blood-brain barrier leakage, edema, and hemorrhage (Asahi et al., 2000, 2001; Lo et al., 2004; Tsuji et al., 2005). Furthermore, interruption of cell-matrix signaling may also trigger anoikis-like processes of brain cell death (Gu et al., 2002; Lee and Lo, 2004). Overall, the overwhelming evidence points to a detrimental effect in the acute phase after brain injury. Hence, there is an emerging rationale for developing MMP inhibitors for neuroprotection (Asahi et al., 2000, 2001; Sumii and Lo, 2002; Gu et al., 2005).

In contrast to these acute neurovascular events, what happens during the delayed stages after brain injury may be quite different. Our present study suggest that, in fact, MMPs may play beneficial roles by mediating the neurogenic response in the SVZ. By allowing neuroblasts to expand and migrate, MMPs should facilitate the endogenous recovery response in a damaged brain. Our immunohistochemistry data suggest that MMP-9 plays a role in this phenomenon. A caveat here is that our MMP inhibitor GM6001 broadly targets the entire MMP family. What other proteases may be involved? A more careful delineation of how the full spectrum of MMP family members and other proteases interact to modulate matrix and subserve neurogenesis is warranted. A second caveat involves the precise mechanisms and consequences of neuroblast migration. It remains possible that the neuroblast migration toward damaged tissue reflects an endogenous response to inflammation. Thus, insofar as MMPs are known to mediate neuroinflammation (Yong et al., 2001; Rosenberg, 2002), suppression of neuroblast profiles in our MMP inhibited brains may simply be attributable to reductions in inflammation and may be unrelated to matrix modulation and migration per se. Although our initial survey of delayed stroke brains show no clear effects of MMP inhibitors on microglial accumulation (supplemental Fig S1, available at www.jneurosci.org as supplemental material), the precise interactions between MMPs, cell-matrix migration, and inflammation warrant additional careful study.

There is no question that neurogenesis is amplified after brain injury. If we wish to augment these endogenous substrates of neurogenesis and recovery to treat brain injury and neurodegeneration, we have to fully understand the molecular events that subserve neuroblast expansion and migration. Our data here show for the first time that extracellular proteolysis via MMP is required for neuroblast migration as the brain attempts to heal itself. Finding ways to modulate these endogenous matrix mechanisms may allow us to ultimately enhance neurogenic recovery after brain injury and disease.

\section{References}

Arvidsson A, Collin T, Kirik D, Lindvall O (2002) Neuronal replacement from endogenous precursors in the adult brain after stroke. Nat Med 8:963-970.
Asahi M, Asahi K, Jung JC, del Zoppo GJ, Fini ME, Lo EH (2000) Role for matrix metalloproteinase 9 after focal cerebral ischemia: effects of gene knockout and enzyme inhibition with BB-94. J Cereb Blood Flow Metab 20:1681-1689.

Asahi M, Wang X, Mori T, Sumii T, Jung JC, Moskowitz MA, Fini ME, Lo EH (2001) Effects of matrix metalloproteinase 9 gene knockout on the proteolysis of blood-brain barrier and white matter components after cerebral ischemia. J Neurosci 21:7724-7732.

Chen J, Zacharek A, Zhang C, Jiang H, Li Y, Roberts C, Lu M, Kapke A, Chopp M (2005) Endothelial nitric oxide synthase regulates brain-derived neurotrophic factor expression and neurogenesis after stroke in mice. J Neurosci 25:2366-2375.

Gasche Y, Fujimura M, Morita-Fujimura Y, Copin JC, Kawase M, Massengale J, Chan PH (1999) Early appearance of activated matrix metalloproteinase-9 after focal cerebral ischemia in mice: a possible role in blood-brain barrier dysfunction. J Cereb Blood Flow Metab 19:1020-1028.

Gross CG (2000) Neurogenesis in the adult brain: death of a dogma. Nat Rev Neurosci 1:67-73.

Gu Z, Kaul M, Yan B, Kridel SJ, Cui J, Strongin A, Smith JW, Liddington RC, Lipton SA (2002) S-nitrosylation of matrix metalloproteinases: signaling pathway to neuronal cell death. Science 297:1186-1190.

Gu Z, Cui J, Brown S, Fridman R, Mobashery S, Strongin AY, Lipton SA (2005) A highly specific inhibitor of matrix metalloproteinase-9 rescues laminin from proteolysis and neurons from apoptosis in transient focal cerebral ischemia. J Neurosci 25:6401-6408.

Heo JH, Lucero J, Abumiya T, Koziol JA, Copeland BR, del Zoppo GJ (1999) Matrix metalloproteinases increase very early during experimental focal cerebral ischemia. J Cereb Blood Flow Metab 19:624-633.

Jin K, Mao XO, Sun Y, Xie L, Greenberg DA (2002) Stem cell factor stimulates neurogenesis in vitro and in vivo. J Clin Invest 110:311-319.

Larsen PH, Wells JE, Stallcup WB, Opdenakker G, Yong VW (2003) Matrix metalloproteinase- 9 facilitates remyelination in part by processing the inhibitory NG2 proteoglycan. J Neurosci 23:11127-11135.

Lee SR, Lo EH (2004) Induction of caspase-mediated cell death by matrix metalloproteinases in cerebral endothelial cells after hypoxia reoxygenation. J Cereb Blood Flow Metab 24:720-727.

Lichtenwalner RJ, Parent JM (2005) Adult neurogenesis and the ischemic forebrain. J Cereb Blood Flow Metab 25:722-729.

Lo EH, Wang X, Cuzner ML (2002) Extracellular proteolysis in brain injury and inflammation: role for plasminogen activators and matrix metalloproteinases. J Neurosci Res 69:1-9.

Lo EH, Broderick JP, Moskowitz MA (2004) tPA and proteolysis in the neurovascular unit. Stroke 35:354-356.

Mun-Bryce S, Rosenberg GA (1998) Matrix metalloproteinases in cerebrovascular disease. J Cereb Blood Flow Metab 18:1163-1172.

Nakatomi H, Kuriu T, Okabe S, Yamamoto S, Hatano O, Kawahara N, Tamura A, Kirino T, Nakafuku M (2002) Regeneration of hippocampal pyramidal neurons after ischemic brain injury by recruitment of endogenous neural progenitors. Cell 110:429-441.

Parent JM, Vexler ZS, Gong S, Derugin N, Ferriero DM (2002) Rat forebrain neurogenesis and striatal neuron replacement after focal stroke. Ann Neurol 52:802-813.

Power C, Henry S, Del Bigio MR, Larsen PH, Corbett D, Imai Y, Yong VW, Peeling J (2003) Intracerebral hemorrhage induces macrophage activation and matrix metalloproteinases. Ann Neurol 53:731-742.

Raber J, Fan Y, Matsumori Y, Liu Z, Weinstein PR, Fike JR, Liu J (2004) Irradiation attenuates neurogenesis and exacerbates ischemia-induced deficits. Ann Neurol 55:381-389.

Reeves TM, Prins ML, Zhu J, Povlishock JT, Phillips LL (2003) Matrix metalloproteinase inhibition alters functional and structural correlates of deafferentation-induced sprouting in the dentate gyrus. J Neurosci 23:10182-10189.

Robin AM, Zhang ZG, Wang L, Zhang RL, Katakowski M, Zhang L, Wang Y, Zhang C, Chopp M (2005) Stromal cell-derived factor lalpha mediates neural progenitor cell motility after focal cerebral ischemia. JCereb Blood Flow Metab 25:744-751.

Rosenberg GA (2002) Matrix metalloproteinases in neuroinflammation. Glia 39:279-291.

Schneider A, Kruger C, Steigleder T, Weber D, Pitzer C, Laage R, Aronowski J, Maurer MH, Gassler N, Mier W, Hasselblatt M, Kollmar R, Schwab S, 
Sommer C, Bach A, Kuhn HG, Schabitz WR (2005) The hematopoietic factor G-CSF is a neuronal ligand that counteracts programmed cell death and drives neurogenesis. J Clin Invest 115:2083-2098.

Sternberg SR (1983) Biomedical image processing. IEEE Comput 16:22-34.

Sumii T, Lo EH (2002) Involvement of matrix metalloproteinase in thrombolysis-associated hemorrhagic transformation after embolic focal ischemia in rats. Stroke 33:831-836.

Sun L, Lee J, Fine HA (2004) Neuronally expressed stem cell factor induces neural stem cell migration to areas of brain injury. J Clin Invest 113:1364-1374.

Tsuji K, Aoki T, Tejima E, Arai K, Lee SR, Atochin DN, Huang PL, Wang X, Montaner J, Lo EH (2005) Tissue plasminogen activator promotes matrix metalloproteinase-9 upregulation after focal cerebral ischemia. Stroke 36:1954-1959.

Wang X, Jung J, Asahi M, Chwang W, Russo L, Moskowitz MA, Dixon CE,
Fini ME, Lo EH (2000) Effects of matrix metalloproteinase-9 gene knock-out on morphological and motor outcomes after traumatic brain injury. J Neurosci 20:7037-7042.

Wang X, Lee SR, Arai K, Lee SR, Tsuji K, Rebeck GW, Lo EH (2003) Lipoprotein receptor-mediated induction of matrix metalloproteinase- 9 by tissue plasminogen activator. Nat Med 9:1313-1317.

Yong VW, Power C, Forsyth P, Edwards DR (2001) Metalloproteinases in biology and pathology of the nervous system. Nat Rev Neurosci 2:502-511.

Zhang R, Zhang Z, Zhang C, Zhang L, Robin A, Wang Y, Lu M, Chopp M (2004) Stroke transiently increases subventricular zone cell division from asymmetric to symmetric and increases neuronal differentiation in the adult rat. J Neurosci 24:5810-5815.

Zhang RL, Zhang ZG, Chopp M (2005) Neurogenesis in the adult ischemic brain: generation, migration, survival, and restorative therapy. Neuroscientist 11:408-416. 